\title{
Psychiatric morbidity among physically ill patients in a Ugandan Regional Referral Hospital
}

\author{
*Rukundo ZG $\mathrm{ZG}^{1}$, Musisi $\mathrm{S}^{2}$, Nakasujja $\mathrm{N}^{2}$
}

1. Department of Psychiatry, Mbarara University of Science and Technology, Mbarara, Uganda

2. Department of Psychiatry, Makerere University, College of Health Sciences, Kampala, Uganda

\begin{abstract}
Background: Mental illness is a global health burden that remains poorly understood even by health care providers. It is important to get insight of the prevalence, clinical features and management of psychiatric morbidity in general practice in Uganda as it affects treatment outcome.

Objective: To determine the prevalence, types and associations of psychiatric morbidity as seen among adult in-patients on medical and surgical wards of Mbarara Regional Referral hospital as a prototype Ugandan regional referral hospital.

Methods: This was a cross sectional descriptive study. Psychiatric diagnosis was arrived at by administering the Mini International Neuropsychiatric Interview (MINI) as the diagnostic instrument.

Results: Of the 258 participants in this study, 109 (42\%) met criteria for at least one DSM IV psychiatric diagnosis. Only 6\% of all the psychiatrically diagnosed patients were recognized by their treating doctors as having mental illness.

Conclusion: The psychiatric disorders on the general medical and surgical wards are highly prevalent and not recognized by staff on these wards despite their common occurrence. There is need for sensitisation of staff on recognition and management of psychiatric disorders in physical illness.

African Health Sciences 2013; 13 (1): 87 - 93 http://dx.doi.org/10.4314/ahs.v13i1.13
\end{abstract}

\section{Introduction}

Physical and mental disorders commonly occur together but the psychiatric disorders are often misunderstood and misdiagnosed. ${ }^{1,2,3}$. Why does it happen like this? The attending physicians usually have a bias to physical illness and have little time for the psychological assessment and management of comorbid psychiatric disorders. Therefore most patients with co-morbid psychiatric disorders go untreated. ${ }^{4}$, 5. Previous studies conducted at Mulago national referral hospital in Uganda have reported similar findings. Nakasujja $(2002)^{6}$ reported a $48 \%$ prevalence of psychiatric morbidity among the elderly patients on the non-psychiatric wards of Mulago hospital. A general psychiatric consultation liaison study by Musisi and colleagues ${ }^{7}$ in the same hospital found that majority of patients with mental illness were neglected, poorly treated and considered to increase burden in patient management on their

\begin{tabular}{|l|}
\hline *Corresponding author: \\
Godfrey Zari Rukundo \\
Department of Psychiatry \\
Mbarara University of Science and Technology \\
P. O. Box 1410 \\
Mbarara, Uganda \\
Tel: (mob)+256772663671 \\
Email:rukundogzari@yahoo.co.uk, \\
gzrukundo@hotmail.com
\end{tabular}

African Health Sciences Vol 13 Issue 1 March 2013 respective admitting wards. After assessment, about $60 \%$ of these patients were successfully treated and psychiatrically discharged from the hospital while still on their respective admitting wards.

The co-occurrence of mental and physical disorders seems to support the mind-body interaction (substance dualism) suggested by Descartes (1641) as described by Nadler S \& Morris $\mathrm{K}$ (1997). ${ }^{8}$ It proposes that although the mind and body are distinctly different, they interact and affect each other. Changes in the body affect the mind and vice versa. Studies have been conducted to look at this relationship. All these studies point to the common co-existence of physical and psychiatric disorders. Hence, as suggested by Clarke and colleagues (1989) ${ }^{9}$, patients should not be assessed for mental disorders after ruling out a physical illness or when the diagnosis is unclear, but should be routinely assessed for mental illness.

Various studies have indicated that presence of a psychiatric disorder in medically ill patients increases the cost of health care due to repeated ineffective use of services, increased length of hospital stay, hospitalization rates and mortality. ${ }^{10,11,12,13}$ It is for this reason that the World Health Organization recommended the integration of mental health services into general health care services. It is hoped that this integration will improve recognition and 
management of mental health problems. The Uganda Ministry of Health has embraced the policy and now integrates mental health into general health services. ${ }^{14,15}$ Little is known about the presentation and prevalence of psychiatric disorders on the general wards in developing countries including Uganda.

Therefore, this cross-sectional quantitative study aimed to investigate the prevalence, types, and associations of psychiatric morbidity among admitted adult patients on medical and surgical wards of a Ugandan regional referral hospital. It was felt that knowing the prevalence of the psychiatric disorders on the medical and surgical wards in Mbarara hospital would provide data that would help in projecting and planning of the newly built regional psychiatric units and as a model for the rest of the regional referral hospitals in Uganda.

\section{Methods}

This was a descriptive cross sectional study conducted to determine the prevalence, types and associations of psychiatric morbidity as seen among adult inpatients on medical and surgical wards of Mbarara Hospital Regional Referral Hospital in South western Uganda. Each participant completed a questionnaire on socio-demographic characteristics and the Mini International Neuropsychiatric Interview (MINI) that was used to determine specific psychiatric diagnoses among the participants. The participants' medical records were reviewed to identify the physical diagnosis and treatments that were given by the attending doctors.

Mbarara hospital is also a teaching hospital for students of human medicine, pharmacy, nursing and medical laboratory science of Mbarara University Medical School. The hospital has two general surgical wards, one emergency surgical ward, two general medical wards, three Obstetric and Gynaecological wards, one paediatric unit and one psychiatric unit. Mbarara hospital has a capacity of 350 beds but is often under pressure to accept large numbers of patients, admitting over 15,000 patients in a year. Medical wards admit an average of about 335 patients per month.

The study was reviewed and approved by the Faculty Research and Ethics Committee Makerere University Faculty of Medicine. Permission was also sought from the Administration of Mbarara regional referral hospital. The review of medical records was done with the patient's informed consent. The attending doctors were informed of patients with psychiatric illness and they were referred to the psychiatric unit for management or the psychiatry team was consulted to review the patients on the parent wards. Patients who were younger than 18 years and those who were severely ill and unable to answer questions were excluded from the study. The interviews were conducted on the medical and surgical wards by two research assistants (a registered mental health nurse and a finalist medical student) that were trained by the first author. Interviews took an average of thirty minutes.

The Mini International Neuropsychiatric Interview (MINI) was used to determine specific psychiatric diagnoses. The MINI is a brief diagnostic structured interview designed to generate positive diagnosis for the 17 major Axis I Psychiatric disorders. ${ }^{16}$ Validation of this instrument in Africa was done in Morocco by Kadri et al (2005) ${ }^{17}$ It was translated into Runyankore-Rukiga (a Bantu language spoken by majority of the people in the South western part of Uganda) and back translated into English to ensure that the original meaning was not lost. The questionnaire was interviewer administered. The patient's medical records were reviewed by the first author to check the physical diagnosis, any psychiatric diagnosis given by the attending doctors, treatments being given, past psychiatric history and number of previous admissions. The statistical package for social sciences (SPSS) version 18 was used for statistical analysis at a precision of $5 \%$, and 95\% confidence interval. Frequencies, mean and correlations were calculated. Using cross tabulations and Pearson's correlation, the different dependent variables associated with psychiatric morbidity were identified.

\section{Results}

\section{Socio-demographics and hospitalizations}

Two hundred fifty eighty (258) adult patients were enrolled into the study. One hundred thirty eight (138) of them were males while 120 were females giving a male: female ratio of 1.2:1. Seventy percent ( $\mathrm{n}=$ 180) of the patients were between 18-40 years of age and only about $10 \%(n=26)$ above 60 years. The mean age of the participants was 37.7 years $(\mathrm{SD}=15.42)$, ranging 18-90 years. Most of the participants had either not gone to school or stopped at primary school level, accounting for $71.7 \%(\mathrm{n}=$ $185)$ of the participants. Fifty eighty $(22.5 \%)$ of the participants were single, 151 (58.5\%) were married, $31(12 \%)$ were widowed, and $18(7 \%)$ had separated with their spouses. 
Fifty nine percent $(\mathrm{n}=152)$ of the participants were on medical wards while $41 \%(n=106)$ were on surgical wards. Of the participants on the medical wards, $49 \%(\mathrm{n}=75)$ were male compared to $60 \%$ $(n=64)$ on the surgical wards. As shown in table 2 , more than two thirds $(n=175)$ of the participants were being hospitalized for the first time in their lives, whereas $43 \%(n=65)$ of the participants on the medical wards and $17 \%(\mathrm{n}=18)$ of those on the surgical wards had been hospitalized in the past. Being admitted on the medical wards was significantly associated with history of previous hospitalization $(\mathrm{t}=4.514, \mathrm{p}=.001)$. The number of children was negatively correlated with psychiatric morbidity ( $\mathrm{p}=.047)$. The patients with more children were less likely to have psychiatric morbidity. There was a positive correlation between psychiatric morbidity on the general wards and number of previous hospitalisations $(\mathrm{p}=.032)$. Those who had psychiatric morbidity were more likely to have had previous hospitalisations than those who were mentally well. Similarly, past psychiatric history was found to be positively correlated with the current psychiatric morbidity $(\mathrm{p}=.004)$. In addition, patients with the diagnosis of HIV/AIDS $(p=.008)$ and Malaria $(\mathrm{p}=.031)$ were more likely to have psychiatric morbidity than those who had other physical diagnoses. The six percent of patients identified by attending staff as having mental illness were all confirmed as having diagnosable mental disorders $(p=.002)$ although the specific diagnoses were different.

Table 1: Socio-demographics and other key factors associated with psychiatric morbidity

\begin{tabular}{lccc}
\hline Variable & Frequency n $(\%)$ & Chi square $\left(\mathbf{X}^{2}\right)$ & P- value \\
\hline Age & & & \\
$18-24$ years & $50(19.4)$ & & \\
$25-40$ years & $130(50.4)$ & 5.276 & \\
$41-60$ years & $52(20.2)$ & & \\
More than 60 years & $26(10.1)$ & & \\
Sex & & & \\
Male & $138(53.5)$ & .052 & \\
Female & $120(46.5)$ & & \\
Religion & & & \\
Catholic & $76(29.5)$ & 6.115 & \\
Anglican & $145(56.2)$ & & \\
Moslem & $22(8.5)$ & & \\
Pentecostal/Born again & $12(4.7)$ & & \\
Other & $3(1.2)$ & & \\
Marital status & & & \\
Single & $58(22.5)$ & & \\
Married/Cohabiting & $151(58.5)$ & & \\
Separated/widowed & $49(19.0)$ & & \\
Education & & & \\
No formal education & $41(15.9)$ & & \\
Primary education & $144(55.8)$ & & \\
Post primary education & $73(28.3)$ & & \\
Number of children & & & \\
None & $57(22.1)$ & & \\
1 - 3 & $93(36.0)$ & & \\
$4-6$ & $54(20.9)$ & & \\
More than 6 & $54(20.9)$ & & \\
\hline
\end{tabular}


Table 2: Other factors associated with psychiatric morbidity

\begin{tabular}{|c|c|c|c|}
\hline $\begin{array}{l}\text { Factors associated with } \\
\text { Pyschiatric morbidity }\end{array}$ & n (\%) & Chi-sqaure & P. value \\
\hline \multicolumn{4}{|c|}{ Number of Hospitalizations } \\
\hline Once & $175(67.8)$ & & \\
\hline Twice or more & $83(32.2)$ & 4.592 & .022 \\
\hline \multicolumn{4}{|l|}{ Ward of admission } \\
\hline Medical ward & $152(58.9)$ & & \\
\hline Surgical ward & $106(41.1)$ & 26.114 & .001 \\
\hline \multicolumn{4}{|l|}{ Past psych history } \\
\hline Yes & $12(4.7)$ & & \\
\hline No & $246(95.3)$ & 8.165 & .005 \\
\hline \multicolumn{4}{|l|}{ HIV diagnosis } \\
\hline Yes & $44(17.1)$ & & \\
\hline No & $214(82.9)$ & 6.960 & .007 \\
\hline \multicolumn{4}{|l|}{ Begnin tumours } \\
\hline Yes & $24(9.3)$ & & \\
\hline No & $234(90.7)$ & 7.704 & .004 \\
\hline \multicolumn{4}{|l|}{ Malaria } \\
\hline Yes & $29(11.2)$ & & \\
\hline No & $229(88.8)$ & 4.630 & .026 \\
\hline \multicolumn{4}{|l|}{ Diabetes/Cardiac disease } \\
\hline Yes & $23(8.9)$ & & \\
\hline No & $235(91.1)$ & .200 & ns \\
\hline
\end{tabular}

Table 3: Frequency of the psychiatric disorders

\begin{tabular}{ll}
\hline Psychiatric disorder & $\begin{array}{l}\text { Prevalence in study } \\
\text { sample (N =258) }\end{array}$ \\
\hline Major depression & $33.7 \%(87)$ \\
Anxiety disorder & $10.9 \%(28)$ \\
Adjustment disorder & $0.8 \%(2)$ \\
Alcohol use disorder & $5.4 \%(14)$ \\
Delirium & $1.6 \%(4)$ \\
Dementia & $0.8 \%(2)$ \\
Organic psychosis & $1.9 \%(5)$ \\
Personality disorder & $0.8 \%(2)$ \\
Other & $1.6 \%(4)$ \\
\hline
\end{tabular}

Psychiatric disorders and their recognition by attending doctors

Of the 258 participants, 109 (42\%) had at least one DSM IV psychiatric diagnosis. Of the 109 participants with psychiatric disorder, $73 \%(\mathrm{n}=80)$ met criteria for one specific psychiatric diagnosis, $24 \%(\mathrm{n}=26)$ met criteria for two psychiatric diagnoses while $3 \%(n=3)$ met criteria for three psychiatric diagnoses.

Depression was found in 34\% ( $n=87)$ of the sample and more than half ( $\mathrm{n}=51$ ) of them had mildmoderate depression, whereas 33\% $(\mathrm{n}=29)$ had severe depression. Only two patients met criteria for bipolar affective disorder, depressive phase. Both patients with bipolar depression were female, on the medical ward, and were included in the overall number of depression. Twenty six percent of the participants with depressive disorders were on surgical wards while $74 \%$ were on medical wards. Two thirds of the participants with anxiety disorders were on medical wards.

Anxiety disorder was the second most prevalent psychiatric disorder (11\%) among the participants with generalized anxiety disorder and panic disorder being the most common and accounting for $75 \%$ of the anxiety disorders. Five percent of the respondents had alcohol use disorder and two patients (aged 40 years) HIV dementia. Two percent of the participants $(n=5)$ had psychotic disorder due to a general medical condition. Of the 109 patients who had MINI psychiatric diagnoses, $12 \mathrm{had}$ attempted or had had a plan for suicide within the past 3 months, while 2 had had death wishes but did not want to commit suicide. Nine of the patients who had attempted suicide had the diagnosis of 
major depression, one had anxiety disorder, and two had alcohol use disorders. Twenty six percent of the participants with depressive disorders were on surgical wards while $74 \%$ were on medical wards. Despite the high prevalence of psychiatric diagnosis, the attending doctors identified only $6 \%(n=7)$ of them and the diagnoses given were depression, psychotic disorder, confusion and suicide attempt. It is important to note that all the seven participants with psychiatric morbidity identified by the attending doctors were on the medical wards. None of the participants on surgical wards were identified by the attending doctors as having a psychiatric disorder.

All the 258 patients that participated in the study were being treated for a physical illness. The most common surgical diagnoses made were bone fractures $(9.7 \%)$ and benign tumors $(4.7 \%)$ while the most common medical diagnoses were HIV/AIDS $(21.7 \%)$, pneumonia $(17.8 \%)$, malaria $(13.2 \%)$ and tuberculosis $(9.7 \%)$. Some of the participants had more than one physical diagnosis. For example, some participants, who had been operated or were to be operated on and admitted on surgical ward, had medical illnesses. Majority of the participants were on more than one medication e.g. analgesics and antibiotics. More than two thirds (69\%) of the participants were on antibiotics while more than half (57\%) of them were on analgesics. Twelve percent were on anti-malarial drugs, and $2 \%$ on Antiretroviral medication.

\section{Discussion}

This study demonstrates high prevalence and poor recognition of psychiatric disorder on the general wards of Mbarara regional referral hospital in Southwestern Uganda. The findings are similar to those at the National referral general hospital (Mulago) in located in the capital city (Kampala) in central region. ${ }^{6,7,18}$ The findings are also similar to what has been found in other parts of the world., ${ }^{2,19}$ The problem of poor recognition and management of mental illness co-occurring with physical illness is not limited to one hospital / region or country but rather a global problem that needs to be addressed. Goldberg $^{20}$ in the United Kingdom, Shyangwa and colleagues $^{21}$ in Nepal, and Murali ${ }^{22}$ have reported similar findings.

Depression has been found to be the mental illness most commonly associated with physical illness. Indeed, the rate of $34 \%$ as shown in this study is quite high and should not be ignored in routine management of patients on the general wards and in planning of services. Anxiety disorders (11\%) and substance use disorders (5.4\%) are also reasonably common on the general wards and may complicate patient care if not recognized and managed accordingly. In the process, the low recognition or misdiagnosis increases expense on care. This does not only frustrate the health workers and the government, but also the patient and the carers. ${ }^{23}$ In addition to frustration, the poor recognition of mental illness among the physically ill can lead to increased morbidity, poor prognosis of the physical illness, mortality, and increased cost of care. This study did not look at these possible complications of poor recognition. Future studies should consider them.

Although some of the symptoms of psychiatric disorders like depression are similar to what is found in physical disorders, various studies have indicated patients with physical illness and mental illness have poorer levels of functioning, morbidity and mortality compared to patients with similar physical disorders but without depression. In this study, psychological effects of being admitted in hospital were not fully differentiated from the psychological distress the patients could have come with to hospital for admission. However, patients were assessed within first week of admission using a diagnostic instrument. We don't expect the hospital admission to have caused so much distress in one week would have led to a new diagnosis. So, we considered psychiatric symptoms to have preceded admission. All participants in the study were being treated for a physical illness. Most patients had not been fully investigated to establish definitive physical diagnosis and the diagnoses given were mainly from history taking and physical examination.

Due to the limited number of beds at Mbarara hospital, only acute cases are given priority, leaving out those with chronic and disabling conditions that may often include mental illnesses. The other reason for poor recognition of mental illness in the general setting is that non-mental health workers may assume that the depressive symptoms are just secondary medical illness. Therefore, little is done about the depressive symptoms per se. As a result, many physically ill patients with depression end up using more health care resources - for example in investigations and medications and ongoing treatment for unresolved problems.

The relationship between medical and psychiatric illness is multifactoral. The physical illness can cause the mental illness but also the mental illness can cause the physical illness. ${ }^{23}$ Drug treatment for 
the physical illness can complicate into mental illness, the two illnesses can coexist and the medical illness can be as a result of the mental illness. Therefore assessing the patient for both illnesses is imperative ${ }^{22,}$ 23, 24 The most common surgical diagnoses made were bone fractures $(9.7 \%)$ and benign tumors $(4.7 \%)$ while the most common medical diagnoses were HIV/AIDS (21.7\%), pneumonia (17.8\%), malaria $(13.2 \%)$ and tuberculosis $(9.7 \%)$. Some respondents had more than one diagnosis. For example, some of the patients on surgical ward also had concurrent medical diagnosis. Physical illnesses associated with disability (e.g. fractures), shame and stigma (e.g. HIV/AIDS and tuberculosis) and long duration of illness (e.g. begnin tumours) were associated with psychiatric morbidity. ${ }^{24,25}$

This study had limitations. Some of the patients admitted on the medical and surgical wards such as those younger than 18 , were excluded from the study yet they could have mental illness symptoms.

\section{Conclusion}

There is high prevalence of psychiatric disorders on the general medical and surgical wards of Mbarara Regional hospital and over 90\% are not recognized by staff on these wards. The picture is not different even at Mulago National Referral hospital. This could be due to orientation to physical symptoms and signs and limited knowledge of psychiatric morbidity on the general wards. There is need for sensitisation of staff on recognition and management of psychiatric disorders in physical illness.

\section{Recommendations}

It is recommended that assessment for psychiatric morbidity should be included in the routine screening interview of every patient on admission, and psychiatric services should be established on the general wards in the regional referral hospitals. More research is needed at the district hospitals and lower health units if integration of mental health service is to be at all levels of health care.

\section{References}

1. Abiodun O A. Mental health and Primary Care in Africa; East African Medical Journal; 67:273-278.

2. Bridges KW, Goldberg DP. Psychiatric illness in in-patients with neurological disorders: Patient's on discussion of emotional problems with neurologists. British Medical Journal, 1990; 289: 656-658
3. Maguire G P, Julier DL, Hawton K E et al. Psychiatric morbidity and referral on two general medical wards: British Medical Journal, 1974; 1: 268-270.

4. Lipowski Z J. Consultation-Liaison Psychiatry: An overview: American Journal of Psychiatry, 1974; 131: $623-630$

5. Lipowsk Z J. (1985) Psychosomatic Medicine and Liaison psychiatry Plenum Publications, New York

6. Nakasujja, N. (2002): Psychiatric disorders among elderly in-patients on non-psychiatric Wards of Mulago hospital. M.Med Thesis, Makerere University Kampala Uganda, page 32-40

7. Musisi S, Tugumisirize J, Kinyanda E. Psychiatric consultation-Liaison at Mulago hospital. Makerere Medical Journal 2001; 35:4-11

8. Nadler S \& Morris K (1997): Descartes' Dualism; Philosophical Books, 38, 3 (July 1997) pp. 157-169. http://www.newdualism.org/papers/ S.Nadler-et-al/Baker-Morris-review.htm

9. Clarke DM, Minas IH, Stuart G. W. The prevalence of psychiatric morbidity in general hospital (Prince Henry's hospital, Melbourne) inpatients: British Journal of Psychiatry, 1989; 154:504-9.

10. Winokur G, Black D W, Nasrallah Depression secondary to medical illnesses and other psychiatric disorders American Journal of Psychiatry, 1988; 145;233-237

11. Steinberg MD, Saravay SM, Weinschel, B., et al Psychological co morbidity and length in a general hospital; American Journal of Psychiatry 1991; 148: 324-329.

12. Abiodun OA, Ogunremi OO. Psychiatric morbidity in medical and surgical wards of a Nigerian general hospital. Journal of Psychosomatic Medicine Res., 1990; 34(4):409-14

13. Lloyd GG (1991). Text book of General Hospital Psychiatry. $1^{\text {st }}$ Edition, Longman Singapore publishers ( Pte) Ltd

14. Uganda Ministry of Health Strategic plan (1999), Government of the Republic of Uganda.

15. Kigozi F. Integrating mental health into primary health care -Uganda's experience. South African Psychiatry Review; 200710:17-19

16. Sheehan D, Janavs J, Baker R, et al (2002): Mini International Neuro-psychiatric Interview Version 5.0.0.

17. Kadri N, Agoub M, Gnaoui, S E, Alami, K.M, Hergueta, T, Moussaoui, D. Morroccan 
colloquial Arabic version of the Mini International Neuropsychiatric Interview (MINI): qualitative and quantitative validation. European Psychiatry, 2005; 20 (2) 193-85.

18. Katorogo JB, (2001): Prevalence of Depressive symptoms among medically ill in-patients; Thesis Diploma in Clinical Mental Health, Butabika Hospital, Ministry of Health, Uganda. Page 2237.

19. Kiganwa A. Psychiatric morbidity and referral rate among medical in-patients at Kenyatta National Hospital. East African Medical Journal, 1991; 68 (5) 383-388.

20. Goldberg D. The detection and treatment of depression in the physically ill. World Psychiatry 2010; 9:16-20
21. Shyangwa PM, Joshi D, Sherchan S, Thapa KB (2009): Psychiatric morbidity among physically ill persons in eastern Nepal

22. Murali R.. Depression in the Physically Ill. Primary Psychiatry. 2008;15 (9):44-50

23. Ndetei DM, KhasakhalaL I, Mutiso V, Mbwayo AW. Suicidality and depression among adult patients admitted in general medical facilities in Kenya. Annals of General Psychiatry 2010; 9:7

24. MacHale S. Managing depression in physical illness. Advances in psychiatric treatment 2002; 8: 297306

25. Kendell RE. The distinction between mental and physical illness. British Journal of psychiatry; 2001; 178, 490-493. 